\title{
Pemphigus in the eastern region of Turkey
}

\author{
Ibrahim Halil Yavuz¹, Goknur Ozaydın Yavuz, Irfan Bayram², Serap Gunes Bilgili
}

${ }^{1}$ Department of Dermatology, Yuzuncu Yil University Faculty of Medicine, Van, Turkey

${ }^{2}$ Department of Pathology, Yuzuncu Yil University Faculty of Medicine, Van, Turkey

Adv Dermatol Allergol 2019; XXXVI (4): 455-460

DOI: https://doi.org/10.5114/ada.2019.87449

\begin{abstract}
Introduction: Pemphigus refers to a group of rare autoimmune vesiculobullous diseases with high morbidity and mortality, mainly affecting the skin and the mucosae.

Aim: To evaluate the incidence of pemphigus in the Eastern region of Turkey by analysing the clinical characteristics of the patients including the drugs used in the treatment and their side-effect profiles and dosing schedules, duration of treatment, length of hospital stay, and the characteristics of the lesions.

Material and methods: The retrospective study included 130 pemphigus patients who were admitted to the inand out-patient dermatology clinics at the University Medical School between January 2005 and October 2017. Age, gender, length of hospital stay, localization of the lesions, initial and maintenance dosages, treatmentrelated complications, comorbidities, family history, and smoking status were recorded for each patient.

Results: The 130 patients included 62 (47.69\%) men and 68 (52.31\%) women, of which 73 (56.15\%) patients had a smoking history. The annual incidence rate of pemphigus was $1.036 / 100,000$ population and pemphigus vulgaris (PV) was the most common clinical subtype of pemphigus in our patients ( $n=122 ; 93.85 \%)$. The most common side effect of the treatment was oral candidiasis, followed by Cushingoid appearance.

Conclusions: The results indicated that the incidence of pemphigus is remarkably high in the Eastern region of Turkey and the associated factors including lifestyle changes should be taken into mind when considering these patients.
\end{abstract}

Key words: pemphigus, smoking, foods.

\section{Introduction}

The word 'pemphigus' takes its root from the Greek word 'pemphig', which literally means pustule [1]. Pemphigus refers to a group of rare autoimmune vesiculobullous diseases with high morbidity and mortality, mainly affecting the skin and the mucosae. Pemphigus vulgaris (PV) is the most common clinical subtype of pemphigus characterized by the presence of immunoglobulin $\mathrm{G}(\mathrm{IgG})$ antibodies that target the ectodomain of desmosomal cadherins, thus resulting in loss of intracellular adhesion between keratinocytes [2]. Pemphigus has been shown to have an incidence of 0.1-0.5/10,000 population with no gender preponderance. Moreover, pemphigus has a peak incidence between the fourth and sixth decades and is rarely seen in children and elderly patients [3]. The bullae in pemphigus easily rupture to form painful crusted erosions. Moreover, Nikolsky's sign is a typical clinical sign of pemphigus [1].

Diagnosis of pemphigus is based on the clinical (flaccid bullae, vesicles, and erosions on the epithelium of mucous membranes and skin), typical histological (epidermal acantholysis), and fluorescent findings (circulating and bound skin antibodies directed against keratinocyte surface), followed by the Tzanck test which involves cytological examination of the material scraped from the floor of a bulla [2].

High-dose corticosteroids are often required in the treatment of pemphigus to achieve remission and to reduce the chronic recurrent course of the disease although death in all forms of pemphigus mostly results from the side effects of steroids. In addition, adjuvant immunosuppressive agents such as cyclophosphamide, azathioprine, and methotrexate can also be required during the course of the treatment [4].

\section{Aim \\ The aim of this study was to evaluate the incidence of pemphigus in the Eastern region of Turkey by analys- ing the clinical characteristics of the patients including}

Address for correspondence: Dr. Ibrahim Halil Yavuz, Department of Dermatology, Faculty of Medicine, Yuzuncu Yil University, 65100 Van, Turkey, phone: +90 05054753361, e-mail: ihalilyavuz@gmail.com

Received: 7.04.2018, accepted: 11.05.2018. 
the drugs used in the treatment and their side-effect profiles and dosing schedules, duration of treatment, length of hospital stay, and the characteristics of the lesions.

\section{Material and methods}

The retrospective study included 130 pemphigus patients who were admitted to the in- and out-patient dermatology clinics at the Medical School between January 2005 and October 2017. This study was approved by the Clinical Ethics and Research Committee of the Yuzuncu Yıl University, Faculty of Medicine (Date: 21.11.2017, Number: 12). Age, gender, length of hospital stay, localization of the lesions, initial and maintenance dosages, treatment-related complications, comorbidities, family history, smoking status, and the intake food suspected of triggering pemphigus were recorded for each patient. Diagnosis of pemphigus was established based on the clinical and histopathologic examinations followed by a direct immunofluorescence test. PV was diagnosed in the presence of flaccid bullae and vesicles on the skin, mucosal erosions, presence of Nikolsky's sign, and typical localizations of the lesions. Additionally, histopathologic examination was performed to analyse the presence and degree of acantholysis and a direct immunofluorescence test was performed to analyse intercellular IgG and C3 deposits (chicken-wire appearance). On the other hand, pemphigus foliaceus (PF) was diagnosed in the presence of squamous, crusted erosions in a seborrheic distribution and subcorneal bullae, whereas pemphigus erythematous was diagnosed in the presence of erythematous squamous plaques in the face and upper chest and serological detection of antinuclear antibodies, IgA pemphigus was diagnosed in the presence of vesicular pustules distributed in annular pattern and presence of IgA deposition in the direct immunofluorescence test, and paraneoplastic pemphigus was diagnosed based on Camisa and Helm's criteria [5].

Control of disease activity was defined as the time when the established lesions began to heal, formation of new lesions ceased, no non-epithelialized ulcerations and erosions were detected, and the Nikolsky's sign turned negative. Remission was defined as the absence of new lesions while the patient was off therapy for a minimum period of 6 months.

\section{Statistical analysis}

Data were analysed using IBM SPSS Statistics for Windows 22.0 (Armonk, NY: IBM Corp.). The normality of distribution was tested using histogram plot and Kolmogorov-Smirnov test. Descriptive statistics were expressed as mean, standard deviation (SD), median, and minimum-maximum values. Pearson's $\chi^{2}$ test and Fisher's Exact Test were used for comparing $2 \times 2$ tables. Nonparametric data were evaluated using Kruskal-Wallis test and ordinal variables were compared using Spearman's correlation coefficient. A $p$-value of $<0.05$ was considered significant.

\section{Results}

The 130 patients included 62 (47.69\%) men and 68 (52.31\%) women. Of the 130 patients, 73 (56.15\%) patients were smoking and 45 (34.62\%) patients had a history of anxiety disorder or depression. When we analysed the patients according to food suspected of triggering pemphigus, we found that $90 \%$ (117/130) of patients had food intake including garlic, onion and leek (Table 1).

The 12-year incidence rate of pemphigus among our patients was $12.437 / 100,000$ population, with an annual incidence rate of $1.036 / 100,000$ population.

Table 1. Patient characteristics

\begin{tabular}{|c|c|c|c|}
\hline Parameter & & $N$ & $\%$ \\
\hline \multirow[t]{2}{*}{ Gender } & Male & 62 & 47.69 \\
\hline & Female & 68 & 52.31 \\
\hline \multirow[t]{3}{*}{ Affected site } & Skin & 8 & 6.15 \\
\hline & Mouth & 23 & 17.69 \\
\hline & Both & 99 & 76.15 \\
\hline \multirow[t]{2}{*}{ Smoking } & No & 57 & 43.85 \\
\hline & Yes & 73 & 56.15 \\
\hline \multirow{2}{*}{$\begin{array}{l}\text { Intake of food suspected of triggering } \\
\text { pemphigus (onion, garlic or leek) }\end{array}$} & No & 13 & 10.00 \\
\hline & Yes & 117 & 90.00 \\
\hline \multirow[t]{2}{*}{ Anxiety disorder or depression } & No & 85 & 65.38 \\
\hline & Yes & 45 & 34.62 \\
\hline
\end{tabular}


Table 2. Distribution of pemphigus subtypes according to mean age and genders

\begin{tabular}{lcccc}
\hline Pemphigus subtype & $\boldsymbol{N}$ & $\%$ & Mean age [years] & Gender \\
\hline Vulgaris & 122 & 93.85 & $51.72 \pm 12.71$ & $56 \mathrm{M} / 66 \mathrm{~F}$ \\
\hline Foliaceus & 4 & 3.08 & $54.75 \pm 18.21$ & $4 \mathrm{M}$ \\
\hline Erythematous & 1 & 0.77 & 77.00 & $1 \mathrm{~F}$ \\
\hline IgA & 1 & 0.77 & 51.00 & $1 \mathrm{M}$ \\
\hline Paraneoplastic & 1 & 0.77 & 73.00 & $1 \mathrm{M}$ \\
\hline Vegetans & 1 & 0.77 & 56.00 & $1 \mathrm{~F}$ \\
\hline Total & 130 & 100.00 & $52.20 \pm 1.96$ & $62 \mathrm{M} / 68 \mathrm{~F}$ \\
\hline
\end{tabular}

$M$ - male, F-female.

Of the 130 patients, 122 (93.85\%) patients were diagnosed with PV, including 56 (46\%) men and 66 (54\%) women with a mean age of $51.72 \pm 12.71$ years. In addition, 4 (3.08\%) patients were diagnosed with PF who had a mean age of $54.75 \pm 18.21$ years, whereas the other pemphigus subtypes were detected in only 1 patient each (Table 2).

The positive DIF findings for pemphigus subtype were as follows:

- Positive DIF for pemphigus vulgaris; 95.9\% (117/122): intercellular, intraepidermal IgG: 84.4\% (103/122), and intercellular, intraepidermal C3 in 89.3\% (109/122),

- Pemphigus foliaceus (4/4): intercellular, intraepidermal IgG in 75\% (3/4), and intercellular, intraepidermal C3 in $73 \%$ (4/4). Interestingly, 100\% (4/4) of the patients showed C3 deposits in the upper levels of the epidermis,

- Pemphigus erythematous (1/1): intercellular, intraepidermal IgG in the patient (1/1) linear C3 deposits at the basement membrane zone in 100\% (1/1),

- IgA pemphigus (1/1): intercellular, intraepidermal IgA deposits in 100\% (1/1) and IgM deposits at the basement membrane zone in $100 \%(1 / 1)$,

- Paraneoplastic pemphigus (1/1): intercellular, intraepidermal lgG in the patient (1/1), intercellular, intraepidermal C3, and linear C3 deposits at the basement membrane zone in $100 \%(1 / 1)$,

- Pemphigus vegetans (1/1): intercellular, intraepidermal IgG: 100\% (1/1) and intercellular, intraepidermal C3 in $100 \%(1 / 1)$.

Overall mean age was $52.20 \pm 12.96$ years, median hospital stay was 3 days, median diagnostic delay was 4 months, median initial steroid dosage was $80 \mathrm{mg}$, median duration of disease was 7 years, and the median length of time to disease control was 20 days (Table 3 ).

Mean duration of disease was $6.93 \pm 3.28$ years in PV patients as opposed to $6.50 \pm 1.73$ years in PF patients (Table 4).

Steroids alone were administered in all PF patients, whereas steroids + azathioprine were used in 56.2\% and steroids alone were used in $23.97 \%$ of the PV patients (Table 5).
The most common side effect of the treatment was oral candidiasis, followed by Cushingoid appearance. Moreover, most of the patients were detected with more than one side effect (Table 6).

\section{Discussion}

The results indicated that the incidence of pemphigus is considerably high in the Eastern region of Turkey. However, although the incidence rate in our study was found to be higher than that of Finland, Saudi Arabia, the Mediterranean Region in Turkey, Iran, and Greece, it was found to be lower than that of Israel. On the other hand, although the incidence rates in Iran and Greece were lower than the incidence rate in our study, they were found to be higher than those reported in the literature, which could be attributed to the geographical proximity of Turkey to Iran and Greece. The high incidence of pemphigus in our study could be explained by the high rate of consanguineous marriages in our region and the predisposition to pemphigus associated with various human leukocyte antigens (HLA) [6-11].

Pemphigus has been shown to have either an equal incidence in men and women or to have a female preponderance. However, Uzun et al. showed that pemphigus has a male preponderance in countries including

Table 3. Mean and median values for age, hospital stay, diagnostic delay, initial steroid dosage, duration of disease, and length of time to disease control

\begin{tabular}{lcc}
\hline Parameter & $\begin{array}{c}\text { Mean or } \\
\text { median }\end{array}$ & $\begin{array}{c}\text { SD or } \\
\text { min.-max. }\end{array}$ \\
\hline Age [years] & 52.20 & \pm 12.96 \\
\hline Hospital stay [days] & 3.00 & $0.00-30.00$ \\
\hline Diagnostic delay [months] & 4.00 & $1.00-75.00$ \\
\hline Initial steroid dosage [mg] & 80.00 & $60.00-100.00$ \\
\hline Duration of disease [years] & 7.00 & $1.00-12.00$ \\
\hline Time to disease control [days] & 20.00 & $15.00-33.00$ \\
\hline
\end{tabular}


Table 4. Distribution of pemphigus subtypes according to mean duration of disease

\begin{tabular}{lccccc}
\hline Pemphigus subtype & \multicolumn{5}{c}{ Duration of disease [years] } \\
\cline { 2 - 6 } & Mean & SD & Median & Min. & Max. \\
\hline Vulgaris & 6.93 & 3.28 & 7.00 & 1.00 & 12.00 \\
\hline Foliaceus & 6.50 & 1.73 & 6.50 & 5.00 & 8.00 \\
\hline Erythematous & 5.00 & - & 5.00 & 5.00 & 5.00 \\
\hline IgA & 5.00 & - & 5.00 & 5.00 & 5.00 \\
\hline Paraneoplastic & 5.00 & - & 5.00 & 5.00 & 5.00 \\
\hline Vegetans & 7.00 & - & 7.00 & 7.00 & 7.00 \\
\hline
\end{tabular}

Kruskal-Wallis test; $p=0.949$.

Table 5. Distribution of pemphigus subtypes according to the drugs used in the treatment

\begin{tabular}{|c|c|c|c|c|c|c|c|c|c|c|c|c|c|c|}
\hline \multirow[t]{3}{*}{ Pemphigus type } & \multicolumn{14}{|c|}{ Drugs } \\
\hline & \multicolumn{2}{|c|}{ Steroid } & \multicolumn{2}{|c|}{ Steroid + AZA } & \multicolumn{2}{|c|}{$\begin{array}{l}\text { Steroid + } \\
\text { MMP }\end{array}$} & \multicolumn{2}{|c|}{$\begin{array}{l}\text { Steroid + AZA } \\
+ \text { MMP }\end{array}$} & \multicolumn{2}{|c|}{$\begin{array}{l}\text { Steroid + AZA } \\
+ \text { IVlg }\end{array}$} & \multicolumn{2}{|c|}{$\begin{array}{l}\text { Steroid + AZA } \\
+ \text { MMP + IVIg }\end{array}$} & \multicolumn{2}{|c|}{$\begin{array}{l}\text { Steroid + AZA } \\
+M M P+C s A\end{array}$} \\
\hline & $n$ & $\%$ & $n$ & $\%$ & $n$ & $\%$ & $n$ & $\%$ & $n$ & $\%$ & $n$ & $\%$ & $n$ & $\%$ \\
\hline Vulgaris & 30 & 24.59 & 68 & 55.73 & 2 & 1.63 & 1 & 0.82 & 14 & 11.47 & 6 & 4.18 & 1 & 0.82 \\
\hline Foliaceus & 4 & 100 & & & & & & & & & & & & \\
\hline Erythematous & 1 & 100 & & & & & & & & & & & & \\
\hline IgA & 1 & 100 & & & & & & & & & & & & \\
\hline Paraneoplastic & & & & & & & 1 & 100 & & & & & & \\
\hline Vegetans & 1 & 100 & & & & & & & & & & & & \\
\hline
\end{tabular}

Table 6. Side effects of the treatment detected in the patients

\begin{tabular}{lcc}
\hline Side effects & $\boldsymbol{N}$ & $\%$ \\
\hline Oral candidiasis & 101 & 77.70 \\
\hline Cushingoid appearance & 89 & 68.46 \\
\hline Weight gain & 71 & 54.61 \\
\hline Striae & 45 & 34.61 \\
\hline Bacterial infection & 41 & 31.53 \\
\hline Excessive hair growth & 33 & 25.38 \\
\hline Osteoporosis & 29 & 22.30 \\
\hline Glaucoma/cataract & 13 & 10.00 \\
\hline Avascular necrosis & 11 & 8.46 \\
\hline
\end{tabular}

Spain and Saudi Arabia [10-14]. On the other hand, autoimmune diseases are seen at high rates in women. In our study, men and women display a close rate, which means autoimmunity is not the sole factor in the aetiology of this disease. Pemphigus has been linked to different HLA antigens in different populations. This association has been mainly with class-II antigens [15]. Therefore, genetic factors may play an important role in this disease.

Pemphigus usually has an onset between the fourth and sixth decades [3]. However, the mean age of onset has been reported to be lower in some countries such as Iran (38 years) and Tunisia (36 years) $[7,14,16]$. In line with the literature, the mean age of onset was 52.2 years in our patients.

Pemphigus vulgaris (PV) is known to be the most common subtype of pemphigus diseases. However, it has been reported that pemphigus erythematous is more common in Finland, PF is more common in Tunisia, and fogo selvagem (endemic pemphigus foliaceus) is more common in Brazil compared to other subtypes [9, 16-18]. In line with the literature, PV was the most common pemphigus subtype diagnosed in our patients. Moreover, the incidence of PV in our study was remarkably higher than those reported in the literature (122/130; 93.85\%).

The bullae seen in PV can be localized in the mucosae or skin, or both [2]. In our study, the bullae were mostly localized in both the mucosae and skin ( $n=99 ; 76.15 \%)$, whereas the bullae localized in the oral mucosa alone were present in $23(17.69 \%)$ patients. 
Uzun et al. reported that painful and resistant oral ulcerations and erosions were one of the most important indicators of pemphigus in their patients and also noted that the disease was limited to oral mucosa in only $7.3 \%$ of the patients [11]. Similarly, these findings were consistent with the findings of our study, which implicates that PV should be considered in the differential diagnosis of the diseases that manifest only with erosions and ulcerations in the oral mucosa.

The literature indicates that the diagnosis of PV is often delayed due to various reasons. Bozdag and Bilgin reported a mean diagnostic delay of 4.8 months in their patients [19]. Similarly, we had a mean diagnostic delay of 4 months in our patients, which implies that direct immunofluorescence biopsy should be kept in mind in order to prevent a diagnostic error in the patients undergoing oral biopsy.

In our patients, the initial steroid dosage was 60$100 \mathrm{mg} /$ day and the dosage was increased gradually in refractory patients. However, in line with the literature, the steroids were not ceased completely in any patient.

Adjuvant immunosuppressive agents are commonly used in the treatment of PV to reduce the dose of steroids and to achieve better control of the disease. These agents mostly include azathioprine, mycophenolate mophetil, methotrexate, chlorambucil, cyclophosphamide, cyclosporine, and rituximab [2, 20]. In our study, azathioprine was the most common agent used in our patients, followed by mycophenolate mophetil, cyclosporine, and intravenous immunoglobulin (IVIg). However, no patient was treated with methotrexate, chlorambucil, cyclophosphamide, and rituximab.

The most common complications caused by pemphigus treatment include oral candidiasis, weight gain, Cushingoid appearance, bacterial infections, gastritis, osteoporosis, avascular necrosis, necrosis, cataract, and glaucoma $[2,19,20]$. In our study, the most common complication was oral candidiasis ( $n=101 ; 77.7 \%)$. Depending on this finding, we consider that the lesions in the oral mucosa in pemphigus patients may not always activate the disease but may result from a yeast infection such as candidiasis.

Pietkiewicz et al. examined pemphigus cases in the area near the wastewater treatment plant in Poland. The study concluded that some chemical factors (mercury, silica dust, pesticides, thiols, amides, phenols) could trigger autoimmunity in pemphigus [21]. Ruocco et al. showed that the intake of aliment rich in thiols such as onion, garlic, and leek may be food suspected of triggering pemphigus [22]. According to a study, molecules containing thiol or drugs of a group of sulfhydryls may be substances suspected of triggering pemphigus, and acantholysis is probably both biochemical and immunologic [23]. Brenner et al. claim that garlic compounds with stable disulfide and thiol groups in their molecule are capable of inducing acantholysis in vitro [24]. A case report showed that a 49-year-old woman with pemphigus got worse because of intense garlic consumption and her disease was controlled following the removal of garlic from the diet [25]. In another case report, a patient with excessive leek consumption showed an increase in oral pemphigus lesions and the oral lesions were controlled by the elimination of this food from the diet. In our study, we found that most of our patients (90\%) had a high intake of food suspected of triggering pemphigus, which indicates that patients with pemphigus should avoid consuming such foods.

Smoking is commonly known to have adverse effects on the recovery of numerous diseases including oral diseases. However, Mehta et al. reported that smoking led to the improvement of oral PV in a patient and Sullivan et al. suggested that smoking has a protective effect in the treatment of pemphigus [26, 27]. Nevertheless, although our patients had a high rate of smoking (73/130; $56.15 \%)$, smoking did not seem to cause any improvement in the mucosal and skin lesions in our patients. Therefore, our findings contradict those reported by Mehta et al. and Sullivan et al. [28].

Our study was limited since it was single-centred, retrospective, and had a relatively small patient series. Moreover, detection of anti-DSG3 and anti-DSG1 autoantibodies by ELISA and immunofluorescence for pemphigus diagnosis is important. These autoantibodies could not be assessed due to lack of technical equipment in our hospital.

\section{Conclusions}

The results indicated that the incidence of pemphigus is remarkably high in the Eastern region of Turkey. Moreover, it was also revealed that the most common subtype of pemphigus is pemphigus vulgaris, there are foods suspected of triggering pemphigus, and smoking increases the incidence of pemphigus. Nevertheless, further prospective, multicentre studies with larger patient series are needed to substantiate our findings.

\section{Conflicts of interest}

The authors declare no conflict of interest.

\section{References}

1. Michailidou EZ, Belazi MA, Markopoulos AK, et al. Epidemiologic survey of pemphigus vulgaris with oral manifestations in northern Greece: retrospective study of 129 patients. Int J Dermatol 2007; 46: 356-61.

2. Pollmann R, Schmidt T, Eming R, et al. Pemphigus: a comprehensive review on pathogenesis, clinical presentation and novel therapeutic approaches. Clin Rev Allergy Immunol 2018; 54: 1-25.

3. Kneisel A, Hertl M. Autoimmune bullous skin diseases. Part 2: diagnosis and therapy. J Dtsch Dermatol Ges 2011; 9: 927-47. 
4. Mahajan VK, Sharma NL, Sharma RC, et al. Twelve-year clinico-therapeutic experience in pemphigus: a retrospective study of 54 cases. Int J Dermatol 2005; 44: 821-7.

5. Camisa C, Helm TN. Paraneoplastic pemphigus is a distinct neoplasia-induced autoimmune disease. Arch Dermato 1993; 129: 883-6.

6. Kyriakis KP, Vareltzidis AG, Tosca AD. Environmental factors influencing the biologic behaviour of patterns of pemphigus vulgaris: epidemiologic approach. Int J Dermatol 1995; 34: 181-5.

7. Salmanpour R, Shahkar H, Namazi MR, et al. Epidemiology of pemphigus in south-western Iran: a 10-year retrospective study (1991-2000). Int J Dermatol 2006; 45: 103-5.

8. Pisanti S, Sharav Y, Kaufman E, et al. Pemphigus vulgaris: incidence in Jews of different ethnic groups, according to age, sex, and initial lesion. Oral Surg 1974; 38: 382-7.

9. Hietanen J, Salo OP. Pemphigus: an epidemiological study of patients treated in Finnish hospitals between 1969 and 1978. Acta Dermatovenereol (Stockh) 1982; 62: 491-6.

10. Tallab T, Joharji H, Bahamdan K, et al. The incidence of pemphigus in the southern region of Saudi Arabia. Int I Dermatol 2001; 40: 570-2.

11. Uzun S, Durdu M, Akman A, et al. Pemphigus in the Mediterranean region of Turkey: a study of 148 cases. Int J Dermatol 2006; 45: 523-8.

12. Coronel-Pérez IM, Rodríguez-Rey EM, Pérez-Bernal AM, et al. Epidemiology of pemphigus in the Hospital Universitario Virgen Macarena, Seville, Spain, 2005-2006. Actas Dermosifiliogr 2009; 100: 121-5.

13. Alcaide-Martín AJ, Gallardo-Pérez MA, Castillo-Muńoz R, et al. Epidemiologic study of 20 cases of pemphigus at Hospital Clínico Universitario Virgen de la Victoria de Málaga, Spain. Actas Dermosifiliogr 2010; 101: 524-33.

14. Kumar KA. Incidence of pemphigus in Thrissur district, south India. Indian J Dermatol Venereol Leprol 2008; 74: 349-51.

15. Gazit E, Loewenthal R. The immunogenetics of pemphigus vulgaris. Autoimmun Rev 2005; 4: 16-20.

16. Bastuji-Garin S, Souissi R, Blum L, et al. Comparative epidemiology of pemphigus in Tunisia and France: unusual incidence of pemphigus foliaceus in young Tunisian women. J Invest Dermatol 1995; 104: 302-5.

17. Ribeiro AM, Alvarez RR, Friedman H, et al.; Cooperative Group osn Fogo Selvagem Research. The profile of fogo selvagem (endemic pemphigus foliaceus) at the University Hospital of Brasilia-Brazil. Epidemiological and clinical considerations. Int J Dermatol 2005; 44: 293-8.

18. Morini JP, Jomaa B, Gorgi Y, et al. Pemphigus foliaceus in young women. An endemic focus in the Sousse area of Tunisia. Arch Dermatol 1993; 129: 69-73.

19. Bozdag K, Bilgin i. Epidemiology of pemphigus in the western region of Turkey: retrospective analysis of 87 patients. Cutan Ocul Toxicol 2012; 31: 280-5.

20. Kanwar AJ, De D. Pemphigus in India. Indian J Dermatol Venereol Leprol 2011; 77: 439-49.

21. Pietkiewicz P, Gornowicz-Porowska J, Bartkiewicz P, et al. Reviewing putative industrial triggering in pemphigus: cluster of pemphigus in the area near the wastewater treatment plant. Adv Dermatol Allergol 2017; 34: 185-91.

22. Ruocco V, Brenner S, Ruocco E. Pemphigus and diet: does a link exist? Int J Dermatol 2001; 40: 161-3.

23. Brenner S, Bialy-Golan A, Ruocco V. Drug-induced pemphigus. Clin Dermatol 1998; 16: 393-7.
24. Brenner S, Ruocco V, Wolf R, et al. Pemphigus and dietary factors. In vitro acantholysis by allyl compounds of the genus Allium. Dermatology 1995; 190: 197-202.

25. Ruocco V, Brenner S, Lombardi ML. A case of diet-related pemphigus. Dermatology 1996; 192: 373-4.

26. Chorzelski TP, Hashimoto T, Jablonska S, et al. Can pemphigus vulgaris be induced by nutritional factors? Eur J Dermatol 1996; 6: 284-6.

27. Mehta JN, Martin AG. A case of pemphigus vulgaris improved by cigarette smoking. Arch Dermatol 2000; 136: 15-7.

28. Sullivan TP, Elgart GW, Kirsner RS. Pemphigus and smoking. Int J Dermatol 2002; 41: 528-30. 